\title{
A novel code-based iterative PIC scheme for multirate $\mathrm{Cl} / \mathrm{MC}-\mathrm{CDMA}$ communication
}

\author{
Mithun Mukherjee ${ }^{*}$ and Preetam Kumar
}

\begin{abstract}
This paper introduces a novel code-based iterative parallel interference cancellation technique (Code-PIC) for the multirate carrier interferometry/multicarrier code division multiple-access (CI/MC-CDMA) system, which supports simultaneous transmission of high and low data rate users. In Code-PIC scheme, multiple-access interference (MAI) for the desired user is estimated based on the projection of subcarrier and subsequent removal of interference from the received signal depending on specific high or low data rate users. Carrier interferometry $(\mathrm{Cl})$ codes are used to minimize the cross-correlation between users, which significantly reduces the multiple-access interference (MAI) for the desired user. The effect of MAI in CI/MC-CDMA is reduced by giving proper phase shift to different set of users. Improved estimation of MAI in Code-PIC results in lower residual interference after interference cancellation. Simulation results show that Code-PIC scheme offers improved BER performance over AWGN and Rayleigh fading channels compared to Block-PIC and Sub-PIC with reduced latency and complexity.
\end{abstract}

\section{Introduction}

Multicarrier code division multiple-access (MC-CDMA) system is a promising technique for high-speed communication system due to robustness against intersymbol interference (ISI) over multipath. The capacity of CDMA in cellular and wireless personal communication systems is limited by multiple-access interference (MAI) due to simultaneous transmission of more than one user. The interference power increases linearly with the number of simultaneous users. To alleviate MAI, several multiuser detection schemes have been proposed in the literature [1]. The conventional detector follows single-user detection (SUD). In SUD, every user is detected separately in the presence of MAI. Performance improvement is observed with multiuser detection (MUD) schemes, where the information about multiple user is used to detect the desired user. Although notable performance gain is obtained with maximum-likelihood (ML) multiuser detector, the complexity of the detector grows exponentially with the number of users. The iterative expectation-maximization (EM) algorithm enables approximating the ML estimate. EM-based joint data detector [2] has excellent multiuser efficiency and is robust against errors in the estimation of the channel

\footnotetext{
* Correspondence: mithun@iitp.ac.in
Department of Electrical Engineering, Indian Institute of Technology Patna,

* Correspondence: mithun@iitp.ac.in
Department of Electrical Engineering, Indian Institute of Technology Patna, Patna, India - 800013
}

parameters. ML approach requires high computational complexity. To mitigate computational complexity, suboptimal MUD like minimum mean-square error (MMSE) has been proposed. A non-linear MMSE multiuser decision-feedback detectors (DFDs) are relatively simple and can perform significantly better than a linear multiuser detector. Multiuser decision-feedback detectors (DFDs) based on the minimum mean-squared error (MMSE) are reported in [3] over multipath. The MMSE adaptive receiver has a much better performance than matched filter receiver with a slightly higher computational complexity. The group pseudo-decorrelator, the group MMSE detector and the pseudo-decorrelating decisionfeedback detector are proposed by Kapur et al. [4].

Considerable performance improvement can be achieved by the use of interference cancellation (IC) technique. Interference cancellation detector removes interference by subtracting estimates of interfering signals from the received signal. Serial interference cancellation (SIC) has been the active area of research due to its lower complexity compared with other multiuser receiver. SIC [5] removes the interference serially. It is expected that bit error rate (BER) performance improves after each iteration stage of iterative SIC. In high-speed data communications, parallel interference cancellation (PIC) [6] is more preferable due to reduced delay. Hardware complexity is one of the main drawbacks of PIC. 
Performance analysis of improved PIC has been reported in [7]. However, if some of users' information is wrongly detected, then the estimated MAI increases the interference power resulting in degraded BER performance for desired user. The error propagation can be minimized when hard decision is replaced by soft decision of received bits. Soft decision-based IC schemes have been proposed by different authors [8-10].

Fast adaptive MMSE/PIC iterative algorithm [11] has been proposed to reduce overhead introduced during the receiver's training period. Least-squares (LS) joint optimization method [12] is presented for estimating the interference cancellation (IC) parameters, the receiver filter and the channel parameters. Lamare et al. proposed a lowcomplexity near-optimal ordering MMSE design criteria [13] for efficient decision-feedback receiver structure along with successive, parallel and iterative interference cancellation structures. Significant performance improvement is obtained with iterative interference cancellation receiver for underloaded CDMA $[9,10,14,15]$.

Non-linear PIC or SIC performs better compared to other MUD in overloaded system. Suboptimum multiuser detection [16] for overloaded systems has been proposed, but with very specific constraints on the signal set. Multistage iterative interference cancellation has been found suitable in overloaded system [17-19]. Recently, iterative multiuser detection with soft IC for multirate MC-CDMA has been proposed in [20].

The effect of MAI that arises from the cross-correlation between different users' code can be minimized by using Carrier Interferometry (CI) codes [21,22]. CI codes provide flexible system capacity [23] with good spectral sharing. CI codes of length $N$ can support $N$ simultaneous users orthogonally. User capacity can be increased up to $2 N$ by adding additional $N$ pseudo-orthogonal users to the existing system [22]. For synchronous CI/ MC-CDMA uplink, threshold PIC (TPIC) and BlockPIC [24] have been designed to provide better performance than conventional PIC scheme. Block-PIC significantly outperforms the conventional PIC with a slight increase in complexity. Single user bound with a $1 \mathrm{~dB}$ off is obtained in Block-PIC at a BER of 1e-03. In [25], subcarrier PIC (Sub-PIC) has been developed for high-capacity $\mathrm{CI} / \mathrm{MC}$-CDMA with variable data rates. Although the system capacity has been increased up to three times (i.e., system capacity $3 N$ ), higher BER restricts real-time data communication.

This paper attempts to improve the performance of multirate CI/MC-CDMA system by a novel code-based iterative PIC (Code-PIC) scheme. Proper phase shifts between different set of users reduce the effect of MAI. We have shown that BER performance of multirate CI/MC-CDMA improves considerably by using subcarrier projection method of the interfering users. Performance for different combination of low and high data rate users is shown over different channel conditions like additive white Gaussian noise (AWGN) and slow-frequency selective Rayleigh fading channel. Performance comparisons with Block-PIC and Sub-PIC are also presented in this work.

The paper is organized as follows: System model of CI/MC-CDMA is discussed in Sections 2, and Section 3 describes iterative interference cancellation receiver. In Section 4, multirate high-capacity system is explained. Code-PIC for different user sets is outlined in Section 5. Simulation results are presented in Section 6. Computational complexities of conventional PIC, Block-PIC, SubPIC and Code-PIC for multirate CI/MC-CDMA system are evaluated in Section 7. Finally, in Section 8, conclusions are drawn.

\section{System model}

This section describes the model of CI/MC-CDMA system considered in the paper. Synchronous CI/MCCDMA system with $K$ users is considered. Each user employs $N$ subcarriers with binary phase-shift keying (BPSK) modulation. CI code [21,22] of length $N$ for $k$ th user $(1 \geq k \geq K)$ corresponds to

$$
\begin{aligned}
\left\{\beta_{k}^{0}, \beta_{k}^{1}, \beta_{k}^{2}, \ldots \beta_{k}^{N-1}\right\} & =\left\{e^{j \Delta \theta_{k}^{0}}, e^{j \Delta \theta_{k}^{1}}, e^{j \Delta \theta_{k}^{2}}, \ldots e^{j \Delta \theta_{k}^{N-1}}\right\} \\
& =\left\{1, e^{j \Delta \theta_{k}}, e^{2 j \Delta \theta_{k}}, \ldots e^{(N-1) j \Delta \theta_{k}}\right\}
\end{aligned}
$$

where

$$
\Delta \theta_{k}= \begin{cases}\frac{2 \pi k}{N} \quad k=1,2, \ldots, N \\ \frac{2 \pi k}{N}+\frac{\pi}{N} k=N+1, N+2, \ldots, 2 N\end{cases}
$$

\subsection{Transmitter}

The transmitted signal corresponding to $n$th data symbol of the kth user is

$$
s_{k}(t)=\sum_{i=0}^{N-1} \sum_{n=1}^{M} a_{k}[n] \exp \left(j\left(2 \pi f_{i} t\right)+i \Delta \theta_{k}\right) \cdot p\left(t-n T_{b}\right)
$$

where $M$ is the number of data symbols per user per frame. $a_{k}[n]$ is $n$th input data symbol of $k$ th user, which is modeled as a sequence of independent and identically distributed (i.i.d.) random variables taking values from \pm 1 with equal probability. $\left\{f_{i}=f_{c}+i \Delta f,(i=0,1,2, \ldots N-1)\right\}$ is the frequency of $i$ th narrow band subcarrier with center frequency $f_{c}$. $\Delta f$ is selected such that orthogonality between carrier frequencies can be maintained. Typically, $\Delta f=1 / T_{b}$ where $T_{b}$ is bit duration of Nyquist pulse shape $p(t)$. The transmitted signal for $\mathrm{K}$ users can be expressed as

$$
S(t)=\sum_{k=1}^{K} s_{k}(t)
$$




\subsection{Channel model}

The channel is modelled as a slowly varying frequency selective Rayleigh fading channel. It is assumed that every user experiences an independent propagation. Each carrier undergoes a flat fading over entire bandwidth. The frequency selectivity over the entire bandwidth results correlated subcarrier. The correlation between $i$ th subcarrier fade and $j$ th subcarrier fade can be modeled as [26]

$$
\rho_{i j}=\frac{1}{1+\left(\left(f_{i}-f_{j}\right) /(\Delta f)_{c}\right)^{2}}
$$

where $(\Delta f)_{c}$ is the coherence bandwidth. Bandwidth of each subcarrier is chosen to be less than $(\Delta f)_{c}$, i.e., $1 / T_{b}$ $\ll(\Delta f)_{c}<B W$, where BW is the total bandwidth of the transmission. For multipath frequency selective channel, we have assumed 4-fold Rayleigh fading [21,24], i.e., $B W /(\Delta f)_{c}=4$.

The transfer function of the channel of the $i$ th subcarrier for $k$ th user is $\xi_{i, k}=\alpha_{i, k} \cdot \exp \left(\beta_{i, k}\right)$, where $\alpha_{i, k}$ and $\beta_{i, k}$ are complex channel gain and carrier phase offset for $i$ th subcarrier of $k$ th user, respectively.

\subsection{Receiver}

The received signal $r(t)$ can be written as

$$
r(t)=\sum_{k=1}^{K} \sum_{i=0}^{N-1} \alpha_{i, k} a_{k}[n] \cdot \exp \left(j\left(2 \pi f_{i} t+i \Delta \theta_{k}+\beta_{i, k}\right)\right) \cdot p\left(t-n T_{b}\right)+\eta(t)
$$

where $\beta_{i, k}$ is random carrier phase offset uniformly distributed over $[0,2 \pi]$ for $k$ th user in $i$ th subcarrier. Rician amplitude distribution can be applied for $\alpha_{i, k}$ in indoor data communication, where line of sight (LOS) components in received signal can be found. Rayleigh fading would be more appropriate in long distance wireless communication where LOS is hardly possible. For channel model, each resolvable multipath component is assumed to follow Rayleigh fading characteristics. The advantage of using orthogonal code vanishes when multipath fading paths are assumed. $\eta(t)$ represents AWGN with zero mean and double-sided power spectral density $\mathbf{N}_{0} / 2$.

The received signal $r(t)$ is projected on $N$ orthogonal subcarriers and is despread using $k$ th user's CI code. The $i$ th subcarrier component of received signal $r(t)$ can be written as

$$
y_{i}=\sqrt{\frac{2}{N_{0} T_{b}}} \int_{0}^{T_{b}} r(t) \exp \left(-j\left(2 \pi f_{i} t\right)\right) \mathrm{d} t
$$

where $y_{i}$ is the projected $N$ orthogonal subcarrier component of the received signal $r(t)$.
The decision variables for $k$ th user at different subcarriers may be expressed as

$$
\mathbf{r}^{k}=\left[r_{0, i t e r}^{k}, r_{1, i t e r}^{k}, \ldots, r_{N-1, i t e r}^{k}\right]
$$

where $r_{i, i t e r}^{k}$ is decision variable for $i$ th subcarrier of $k$ th user at iter-th iteration stage.

$$
\begin{aligned}
r_{i, i t e r}^{k}=\alpha_{i, k}^{*} \cdot \exp \left(-j\left(i \Delta \theta_{k}\right)\right) \gamma_{i} \\
\quad+\sum_{m=1, m \neq k}^{K} \sqrt{\frac{2 E_{b}}{N_{0}}} \hat{a}_{m}^{(i t e r-1)} \alpha_{i, k}^{*} \alpha_{i, m} \exp \left[j\left(i\left(\Delta \theta_{m}-\Delta \theta_{k}\right)+\left(\hat{\beta}_{i, m}-\beta_{i, k}\right)\right)\right] \\
+\eta_{i} \exp \left(-j\left(i \Delta \theta_{k}\right)\right)
\end{aligned}
$$

where * denotes the complex conjugate and $\eta_{i}$ is Gaussian random variable with zero mean and variance of $\mathbf{N}_{0} / 2 . E_{b}$ is the transmitted bit energy and $\hat{a}_{k}^{(i t e r)}$ is the estimated data of $k$ th user at iter-th iteration stage. $\hat{\beta}_{i, m}$ is the estimate of the phase for $i$ th subcarrier of $m$ th user. For synchronous transmission, $\hat{\beta}_{i, m}=\beta_{i, k}$ is assumed. Further, it is assumed that the received power of every user is same.

When $y_{i}$ is multiplied by $k$ th user's spreading code,

$$
\begin{aligned}
\mathbf{X}_{k} & =\sum_{i=0}^{N-1} y_{i} \exp \left(-j\left(i \Delta \theta_{k}\right)\right) \\
& =\sqrt{\frac{2 E_{b}}{N_{0}}} a_{k}[n]+\sum_{i=0}^{N-1} \sum_{m=1, m \neq k}^{K} \sqrt{\frac{2 E_{b}}{N_{0}}} a_{m} \exp \left[j\left(i\left(\Delta \theta_{m}-\Delta \theta_{k}\right)\right)\right]+\sum_{i=0}^{N-1} \eta_{i} \exp \left(-j\left(i \Delta \theta_{k}\right)\right)
\end{aligned}
$$

Taking the real part of $\mathbf{X}_{k}$

$$
\mathbf{Y}_{k}=\sqrt{\frac{2 E_{b}}{N_{0}}} a_{k}[n]+\mathbf{I}_{k}+\mathbf{N}_{k}
$$

where

$$
\begin{aligned}
& \mathbf{Y}_{k}=\Re\left[\mathbf{X}_{k}\right]=\Re\left[\sum_{i=0}^{N-1} y_{i} \exp \left(-j\left(i \Delta \theta_{k}\right)\right)\right] \\
& \mathbf{I}_{k}=\Re\left[\sum_{i=0}^{N-1} \sum_{m=1, m \neq k}^{K} \sqrt{\frac{2 E_{b}}{N_{0}}} \hat{a}_{m} \exp \left[j\left(i\left(\Delta \theta_{m}-\Delta \theta_{k}\right)\right)\right]\right] \\
& \mathbf{N}_{k}=\Re\left[\sum_{i=0}^{N-1} \eta_{i} \exp \left(-j\left(i \Delta \theta_{k}\right)\right)\right]
\end{aligned}
$$

$\mathbf{I}_{k}$ is the MAI experienced by $k$ th user due to $(K-1)$ users. Multiplication of noise $\left(\eta_{i}\right)$ by the user's spreading code $\left(\exp \left(-j\left(i \Delta \theta_{k}\right)\right)\right)$ does not change the noise distribution. So, additive noise term $\mathbf{N}_{k}$ is zero mean Gaussian random variable with variance of $\mathbf{N}_{0} / 2$ for $k$ th user. 
The average bit error probability for $k$ th user is given by

$$
\begin{aligned}
P_{k}(e) & =\frac{1}{2} \operatorname{Pr}\left\{\mathbf{Y}_{k}>0 \mid a_{a_{k}[n]=-1}\right\}+\frac{1}{2} \operatorname{Pr}\left\{\mathbf{Y}_{k}<\left.0\right|_{a_{k}[n]=1}\right\} \\
& =\operatorname{Pr}\left\{\mathbf{Y}_{k}>0 \mid a_{a_{k}[n]=-1}\right\} \\
& =\operatorname{Pr}\left\{\left(-\sqrt{\frac{2 E_{b}}{N_{0}}}+\mathbf{I}_{k}+\mathbf{N}_{k}\right)>0\right\} \\
& =\operatorname{Pr}\left\{\left(\mathbf{I}_{k}+\mathbf{N}_{k}\right)>\sqrt{\frac{2 E_{b}}{N_{0}}}\right\}
\end{aligned}
$$

The average BER of all users is given by

$$
P(e)=\frac{1}{K} \sum_{k=1}^{K} P_{k}(e)
$$

From the Equation (15), it is clear that if probability of noise and interference term is higher than $\sqrt{\frac{2 E_{b}}{N_{0}}}$, then BER tends to increase. So, cancellation of interference is necessary to obtain a lower bit error probability. This motivates the need for interference cancellation technique.

\section{Iterative interference cancellation receiver}

In this section, conventional PIC structure is discussed. The estimated interference due to $(K-1)$ users is directly subtracted from $r(t)$ for the desired $k$ th user. The improved received signal $\hat{r}_{k}^{\text {iter }}(t)$ of $k$ th user may be written as

$$
\hat{r}_{k}^{i t e r}(t)=r(t)-\sum_{m=1, m \neq k}^{K} \hat{s}_{m}^{i t e r}(t)
$$

where $\hat{s}_{m}^{\text {iter }}(t)$ is the estimated signal at iter-th iteration for the $m$ th user. $\hat{s}_{m}^{i t e r}(t)$ can be written as,

$$
\hat{s}_{m}^{\text {iter }}(t)=\sum_{i=0}^{N-1} \hat{a}_{m}^{\text {iter }-1} \exp \left[j\left(i\left(\Delta \theta_{m}+2 \pi f_{i} t\right)\right)\right]
$$

\subsection{Subcarrier PIC (Sub-PIC)}

In Sub-PIC, the received signal is projected on $N$ orthogonal subcarrier, and the interference due to other users is subtracted at subcarrier level. Using Equations (7) and (17), the received signal of $k$ th user after orthogonal projection is given as:

$$
\begin{aligned}
& \hat{\gamma}_{i}=\sqrt{\frac{2}{N_{0} T_{b}}} \int_{0}^{T_{b}} \hat{i}_{k}^{\text {iter }}(t) \exp \left(-j\left(2 \pi f_{i} t\right)\right) \mathrm{d} t \\
& =\sqrt{\frac{2}{N_{0} T_{b}}} \int_{0}^{T_{b}}\left[r(t)-\sum_{m=1, m \in k}^{K} \hat{s}_{m}^{\text {iter }}(t)\right]\left(\exp \left(-j\left(2 \pi f_{i} t\right)\right) \mathrm{d} t\right. \\
& =\sqrt{\frac{2}{N_{0} T_{b}}} \int_{0}^{T_{T_{0}}}\left[r(t)-\sum_{m=1, m, m p}^{K} \hat{a}_{m}^{i e r-1} \exp \left[j\left(i\left(\Delta \theta_{m}+2 \pi f_{i} t\right)\right)\right]\right]\left(\exp \left(-j\left(2 \pi f_{i} t\right)\right)\right) \mathrm{d} t \\
& =y_{i}-\sum_{m=1, m, k}^{K} \sqrt{\frac{2 E_{b}}{N_{0}} \hat{a}_{m}^{\text {iter }}} \exp \left[j\left(i\left(\Delta \theta_{m}\right)\right)\right]
\end{aligned}
$$

where $\hat{y}_{i}$ is the projected $N$ orthogonal subcarrier component of $\hat{r}_{k}^{i t e r}(t)$. When $\hat{\gamma}_{i}$ is multiplied by $k$ th user's spreading code,

$$
\begin{aligned}
\hat{\mathbf{X}}_{k}^{\text {iter }} & =\sum_{i=0}^{N-1} \exp \left(-j\left(i \Delta \theta_{k}\right)\right) \hat{y}_{i} \\
& =\sum_{i=0}^{N-1} \exp \left(-j\left(i \Delta \theta_{k}\right)\right) y_{i} \\
& -\sum_{i=0}^{N-1} \sum_{m=1, m \neq k}^{K} \sqrt{\frac{2 E_{b}}{N_{0}}} \hat{a}_{m}^{\text {iter }-1} \exp \left[j\left(i\left(\Delta \theta_{m}-\Delta \theta_{k}\right)\right)\right] \\
& =\sqrt{\frac{2 E_{b}}{N_{0}}} a_{k}[n]+\sum_{i=0}^{N-1} \sum_{m=1, m \neq k}^{K} \sqrt{\frac{2 E_{b}}{N_{0}}} a_{m} \exp \left[j\left(i\left(\Delta \theta_{m}-\Delta \theta_{k}\right)\right)\right] \\
& +\sum_{i=0}^{N-1} \eta_{i} \exp \left(-j\left(i \Delta \theta_{k}\right)\right) \\
& -\sum_{i=0}^{N-1} \sum_{m=1, m \neq k}^{K} \sqrt{\frac{2 E_{b}}{N_{0}}} \hat{a}_{m}^{\text {iter }-1} \exp \left[j\left(i\left(\Delta \theta_{m}-\Delta \theta_{k}\right)\right)\right]
\end{aligned}
$$

Taking the real part of $\hat{\mathbf{X}}_{k}^{i t e r}$,

$$
\begin{aligned}
\hat{\mathbf{Z}}_{k}^{i t e r} & =\Re\left[\hat{\mathbf{X}}_{k}^{i t e r}\right] \\
& =\mathbf{Y}_{k}-\hat{\mathbf{I}}_{k}^{i t e r}
\end{aligned}
$$

where $\hat{\mathbf{I}}_{k}^{i t e r}$ is the estimated MAI experienced by $k$ th user due to $(K-1)$ users at iter-th iteration.

$$
\hat{\mathbf{I}}_{k}^{\text {iter }}=\Re\left[\sum_{i=0}^{N-1} \sum_{m=1, m \neq k}^{K} \sqrt{\frac{2 E_{b}}{N_{0}}} \hat{a}_{m}^{\text {iter }-1} \exp \left[j\left(i\left(\Delta \theta_{m}-\Delta \theta_{k}\right)\right)\right]\right]
$$

So, received data of $k$ th user at iter-th iteration can be given as

$$
\begin{aligned}
& \hat{\mathbf{Z}}_{k}^{i t e r}=\mathbf{Y}_{k}-\hat{\mathbf{I}}_{k}^{i t e r} \\
& =\sqrt{\frac{2 E_{b}}{N_{0}}} a_{k}[n]+\mathbf{I}_{k}+\mathbf{N}_{k}-\hat{\mathbf{I}}_{k}^{i t e r}
\end{aligned}
$$

The average bit error probability in Sub-PIC for $k$ th user is given by

$$
\begin{aligned}
P_{k}(e) & =\frac{1}{2} \operatorname{Pr}\left\{\hat{\mathbf{Z}}_{k}^{\text {iter }}>\left.0\right|_{a_{k}[n]=-1}\right\}+\frac{1}{2} \operatorname{Pr}\left\{\hat{\mathbf{Z}}_{k}^{\text {iter }}<\left.0\right|_{a_{k}[n]=1}\right\} \\
& =\operatorname{Pr}\left\{\hat{\mathbf{Z}}_{k}^{\text {iter }}>\left.0\right|_{a_{k}[n]=-1}\right\} \\
& =\operatorname{Pr}\left\{\left(-\sqrt{\frac{2 E_{b}}{N_{0}}}+\mathbf{I}_{k}+\mathbf{N}_{k}-\hat{\mathbf{I}}_{k}^{\text {iter }}\right)>0\right\} \\
& =\operatorname{Pr}\left\{\left(\mathbf{I}_{k}-\hat{\mathbf{I}}_{k}^{\text {iter }}+\mathbf{N}_{k}\right)>\sqrt{\frac{2 E_{b}}{N_{0}}}\right\}
\end{aligned}
$$

The interference term is reduced by the cancellation of estimated interference. From the above Equation (24), it is clear that the bit error probability becomes low in 
Sub-PIC scheme compared to error probability in case of simple matched filter output (Equation (15)).

Again, $\hat{\mathbf{Z}}_{k}^{i t e r}$ can be written as

$$
\hat{\mathbf{Z}}_{k}^{i t e r}=\sqrt{\frac{2 E_{b}}{N_{0}}} a_{k}[n]+\mathbf{W}_{k}^{i t e r}+\mathbf{N}_{k}
$$

where

$$
\mathbf{W}_{k}^{i t e r}=\mathbf{I}_{k}-\hat{\mathbf{I}}_{k}^{i t e r}
$$

The term $\mathbf{W}_{k}^{\text {iter }}$ stands for the residual or uncancelled interference that arises due to imperfect cancellation. In iterative receiver structure, $\mathbf{W}_{k}^{i t e r}$ is reduced after every iteration stages. For initial estimations, after forming the decision variables $\mathbf{r}^{k}$, minimum mean-square error combiner (MMSEC) is employed to make decision in an AWGN channel [27]. Also, in slow-frequency selective channel, the performance of MMSEC is a good solution [28]. MMSEC exploits diversity of frequency selective channel to minimize intercarrier interference (ICI). $\mathbf{Y}_{k}$ can be written as $\mathbf{Y}_{k}=\mathbf{r}^{k} \bar{\omega}$ for $\hat{a}_{k}^{0}[n]$, where $\bar{\omega}$ is the weight vector of the combiner [27]. The decision of $k$ th user at iter $^{\text {th }}$ iteration becomes

$$
\begin{gathered}
\hat{a}_{k}^{i t e r}[n] \cong \operatorname{sgn}\left\{\hat{\mathbf{Z}}_{k}^{i t e r}\right\} \\
\hat{a}_{k}^{0}[n]=\operatorname{sgn}\left\{\mathbf{Y}_{k}\right\}
\end{gathered}
$$

The scheme represented by Equation (27) is referred as hard decision PIC (HDSub-PIC) [25]. The BER performance of Sub-PIC improves significantly by taking soft estimation of the interfering users. In soft decision Sub-PIC (SDSub-PIC), the estimation of the received data is performed by taking soft decisions using non-linear function [17]. The soft decision of $\mathbf{X}_{k}$ is given by $\tilde{\mathbf{x}}_{k}=\phi\left(\mathbf{Y}_{k}-\hat{\mathbf{I}}_{k}^{\text {iter }}\right)$, where $\varphi(x)$ is the non-linear function. Different types of non-linearities like dead-zone non-linearities, hyperbolic tangent and piecewise linear approximation of hyperbolic tangent can be used for $\varphi\{(x)\}$.

i. Dead-Zone Nonlinearity:

$$
\phi(x)= \begin{cases}\operatorname{sgn}(x) & |x| \geq \lambda \\ 0 & |x|<\lambda\end{cases}
$$

If $\lambda=0$ then it becomes similar to hard decisionbased estimation in Equation (27).

ii. Hyperbolic Tangent:

$$
\phi(x)= \begin{cases}\operatorname{sgn}(x) & |x| \geq \lambda \\ \tanh (x / \lambda) & |x|<\lambda\end{cases}
$$

iii. Piecewise linear approximation of Hyperbolic Tangent: In piecewise linear approximation, for all iteration the function $\varphi\{(x)\}$ can be written as

$$
\phi(x)= \begin{cases}\operatorname{sgn}(x)|x| \geq \lambda \\ x / \lambda & |x|<\lambda\end{cases}
$$

The non-linear parameter $\lambda$ is selected such that minimum BER can be obtained for iterative IC process. Here, in SDSub-PIC technique, we have considered piecewise linear approximation of hyperbolic tangent as a non-linear function of soft decision IC process. In the last stage of iteration, the final decision is made by hard detector, $\hat{a}_{k}[n]=\operatorname{sgn}\left\{\mathbf{Y}_{k}-\hat{\mathbf{I}}_{k}^{i t e r}\right\}$. In the next section, multirate high-capacity $\mathrm{CI} / \mathrm{MC}$-CDMA with $3 \mathrm{~N}$ users system is discussed.

\section{Multirate high-capacity $\mathbf{3 N}$ system}

In CI/MC-CDMA system described in Section 2, $N$ length $\mathrm{CI}$ codes support $N$ orthogonal users and additional $N$ users are added by pseudo-orthogonal $\mathrm{CI}$ codes $[21,22]$. To support more users, a high-capacity CI/MCCDMA system is proposed in [29], where the capacity is increased up to $3 N$ users through the splitting of pseudo-orthogonal CI (PO-CI) codes. As defined earlier, the $\mathrm{CI}$ code for $k$ th user $(1<=k<=K)$ is given by $\left[1, e^{j \Delta \theta_{k}}, e^{2 j \Delta \theta_{k}}, \ldots, e^{(N-1) j \Delta \theta_{k}}\right]$. This code is divided into odd and even parts. Further, orthogonal subcarriers are also divided into odd and even parts. The odd/even partitioning of PO-CI and odd/even separation of available subcarriers are useful in adding extra users and hence the system capacity.

In multimedia communication, users transmit at variable data rate. In this paper, different data rate users are broadly grouped into high data rate users (HDR) and low data rate users (LoDR). HDR users are assigned by $N$ contiguous subcarriers. Non-orthogonal odd/even subcarriers with odd/even CI code are allocated to LoDR users. In multipath fading channel, if some of the subcarriers are passed through deep fade, then other subcarriers are used to ensure low BER. The non-contiguous odd-even subcarrier allocation ensures better performance in deep fade as compared to contiguous subcarrier allocation. Proper user allocation algorithm [29] is maintained to minimize the cross-correlation between different user sets. In multirate high-capacity system model, there are five user sets.

$U_{1}$ : assigned normal $\mathrm{CI}$; transmit through all subcarriers

$U_{2}$ : assigned odd CI codes; transmit through odd subcarriers

$U_{3}$ : assigned even $\mathrm{CI}$ codes; transmit through odd subcarriers 
$U_{4}$ : assigned odd CI codes; transmit through even subcarriers

$U_{5}$ : assigned even $\mathrm{CI}$ codes; transmit through even subcarriers

The transmitted signal for multirate high-capacity system can be expressed as

$$
\begin{aligned}
& S(t)=\sum_{k=0}^{N-1} \sum_{i=0}^{N-1} a_{k}[n] \cdot e^{j\left(2 \pi f_{i} t+\frac{2 \pi}{N} \cdot i \cdot k\right)} \cdot p\left(t-n T_{b}\right) \\
& +\sum_{k=N}^{(3 N / 2)-1} \sum_{i=0 \forall i=\mathrm{odd}}^{N-1} a_{k}[n] \cdot e^{j\left(2 \pi f_{i} t+\frac{2 \pi}{N} \cdot i \cdot k+i \Delta \Phi_{1}\right)} \cdot p\left(t-q . n T_{b}\right) \\
& +\sum_{k=3 N / 2}^{2 N-1} \sum_{i=0 \forall i=\text { odd }}^{N-1} a_{k}[n] e^{j\left(2 \pi f i t+\frac{2 \pi}{N} \cdot(i+1) \cdot k+k i \Delta \Phi_{2}\right)} \cdot p\left(t-q . n T_{b}\right) \\
& +\sum_{k=2 N}^{(5 N / 2)-1} \sum_{i=0 \forall i=\text { even }}^{N-1} a_{k}[n] e^{j\left(2 \pi f_{i} t+\frac{2 \pi}{N} \cdot i k+i \Delta \Phi_{3}\right)} \cdot p\left(t-q . n T_{b}\right) \\
& +\sum_{k=5 N / 2}^{3 N-1} \sum_{i=0 \forall i=e v e n}^{N-1} a_{k}[n] \cdot e^{j\left(2 \pi f_{i} t+\frac{2 \pi}{N} \cdot(i+1) \cdot k+k+\Delta \Phi_{4}\right)} \cdot p\left(t-q \cdot n T_{b}\right)
\end{aligned}
$$

It is assumed that HDR users transmit data at ' $\mathrm{q}$ ' times higher than LoDR users. The angles $\Delta \Phi_{1}, \Delta \Phi_{2}$, $\Delta \Phi_{3}$ and $\Delta \Phi_{4}$ are phase shift for the different LoDR sets $\left(U_{i}, i=2,3,4,5\right)$ with respect to HDR users assigned by normal CI codes. Different angles are shown in Figure 1.

$$
\begin{aligned}
\Delta \Phi_{1} & =\pi / 2 \\
\Delta \Phi_{2} & =-\pi / 2 \\
\Delta \Phi_{3} & =-(\pi+\pi / N) \\
\Delta \Phi_{4} & =-\pi / N
\end{aligned}
$$

These phase angles are chosen such that the interferences between different sets is reduced. Let us assume that $R_{1,2}(j, k)$ represents the cross-correlation between $j$ th user in group 1 and $k$ th user in group 2 .

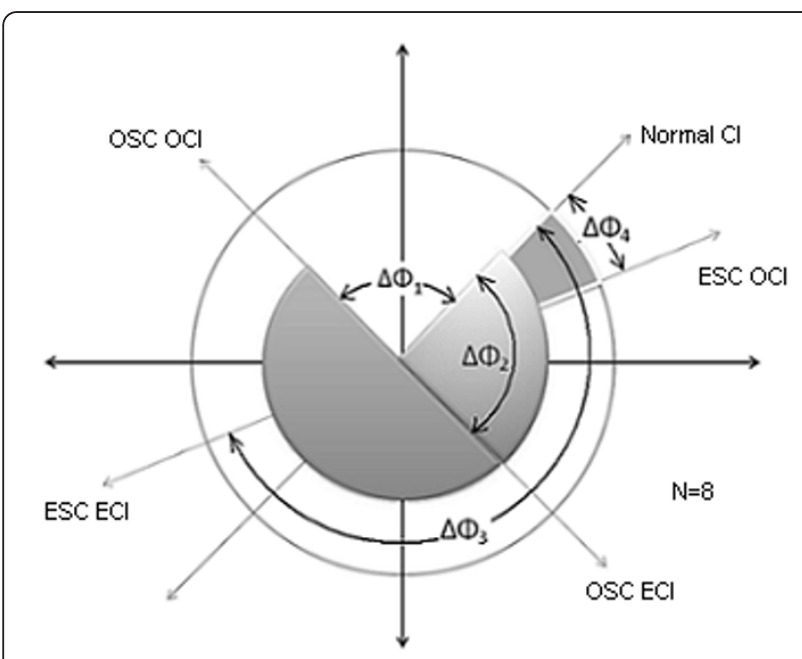

Figure 1 Phase shift between different user sets

$$
R_{1,2}(j, k)=\frac{1}{2 \Delta f} \sum_{i=0}^{N-1} \cos \left[i\left(\Delta \theta_{j}-\Delta \theta_{k}\right)\right]
$$

Here, the cross-correlation between $j$ th user in orthogonal group 1 and all the users in group 2 is identical to the cross-correlation between $(j+1)$ th user in orthogonal group 1 and all the users in group 2. The total numbers of users in group 1 and group 2 are $K_{1}$ and $K_{2}$, respectively.

Let $R_{1,2}(j)$ is the total cross-correlation between $j$ th user and all the users in group 2 .

$$
\begin{aligned}
& R_{1,2}(j)=\frac{1}{K_{2}} \sum_{k=1}^{K_{2}} R_{1,2}(j, k), \ldots \quad \text { for } j \text { th user } \\
& R_{1,2}(j+1)=\frac{1}{K_{2}} \sum_{k=1}^{K_{2}} R_{1,2}(j+1, k), \ldots \quad \text { for }(j+1) \text { th user }
\end{aligned}
$$

In CI-based system, $R_{1,2}(j)=R_{1,2}(j+1)$, i.e., every user in one set has same total cross-correlation from users of the other set. If both sets have same number of users, i. e., $K_{1}=K_{2}$, then the total cross-correlation between $j$ th user in orthogonal group 1 and all the users in group 2 is identical to the cross-correlation between $k^{\prime}$ th user in orthogonal group 2 and all the users in group 1. Total cross-correlation between group 1 and group 2 can be written as

$$
R_{1,2}=\left[\frac{1}{K_{1} \times K_{2}} \sum_{j=1}^{K_{1}} \sum_{k=1}^{K_{2}}\left(R_{1,2}(j, k)\right)^{2}\right]^{\frac{1}{2}}
$$

If $K_{1}=K_{2}=N$, then $R_{1,2}$ becomes

$$
R_{1,2}=\frac{1}{N}\left[\sum_{j=1}^{N}\left(R_{1,2}(j, 0)\right)^{2}\right]^{\frac{1}{2}}
$$

Let $R_{U_{x}, U_{y}}(j, k)$ refers to cross-correlation between $j$ th spreading sequence in $U_{x}$ user set and $k$ th spreading sequence in $U_{y}$ user set. For real signal, the expression is

$$
\begin{aligned}
R_{U_{x}, U_{y}}(j, k) & =\frac{1}{2 \Delta f} \sum_{i=0}^{N-1} \cos \left[i\left(\Delta \theta_{j}-\Delta \theta_{k}\right)\right] \\
& =\frac{1}{2 \Delta f} \sum_{i=0}^{N-1} \cos \left[i\left(\frac{2 \pi}{N} j-\frac{2 \pi}{N} k\right)\right] \\
R_{U_{1}, U_{2}}(j, k) & =\frac{1}{2 \Delta f} \sum_{i=0 \forall i=\text { odd }}^{N-1} \cos \left[i\left(\Delta \theta_{j}-\Delta \theta_{k}\right)\right]
\end{aligned}
$$


Total cross-correlation between $j$ th user and all the user of $U_{2}$ set becomes

$$
R_{U_{1}, U_{2}}(j)=\frac{1}{K_{U_{2}}} \sum_{k=1}^{K_{U_{2}}} R_{U_{1}, U_{2}}(j, k)
$$

where $K_{U_{x}}$ represents total number of users in $U_{x}$ set. In general,

$$
\begin{aligned}
& R_{U_{1}, U_{m}}(j)=\frac{1}{K_{U_{m}}} \sum_{k=1}^{K_{U_{m}}} R_{U_{1}, U_{2}}(j, k), \quad m \in 2,3,4,5 \\
& R_{U_{1}, U_{m}}(j, k)=\frac{1}{2 \Delta f} \sum_{i=0 \forall i=\text { odd }}^{N-1} \cos \left[i\left(\Delta \theta_{j}-\Delta \theta_{k}\right)\right], \quad m \in 2,3
\end{aligned}
$$

and

$$
R_{U_{1}, U_{m}}(j, k)=\frac{1}{2 \Delta f} \sum_{i=0 \forall i=\text { even }}^{N-1} \cos \left[i\left(\Delta \theta_{j}-\Delta \theta_{k}\right)\right], \quad m \in 4,5
$$

So, total cross-correlation between $j$ th user in $U_{1}$ set and all the users in other set is given by

$$
R_{U_{1},\left(U_{2}, U_{3}, U_{4}, U_{5}\right)}(j)=\sqrt{R_{U_{1}, U_{2}}^{2}(j)+R_{U_{1}, U_{3}}^{2}(j)+R_{U_{1}, U_{4}}^{2}(j)+R_{U_{1}, U_{5}}^{2}(j)}
$$

From Equation (44), it is clear that the users of the same set of subcarrier used by $U_{1}$ user set create interference to the $j$ th user of $U_{1}$. set. Assuming orthogonality is maintained in subcarrier, there is no crosscorrelation between $\left[U_{2}, U_{4}\right]$ set and $\left[U_{2}, U_{5}\right]$ set. $U_{2}$ and $U_{3}$ user sets are using different set of subcarriers that is utilized by $U_{4}$ and/or $U_{5}$ sets. In same subcarriers, the cross-correlation between two different user set is minimized by proper phase separation described in Equation (32). For $U_{2}$ user set, all users from $U_{1}$ set and $U_{3}$ user create interference on odd subcarrier. Then, total interference for $j$ th user in $U_{2}$ user is obtained by

$$
R_{U_{2},\left(U_{1}, U_{3}\right)}(j)=\sqrt{R_{U_{2}, U_{1}}^{2}(j)+R_{U_{2}, U_{3}}^{2}(j)}
$$

In multipath channel, intercarrier interference (ICI) occurs due to non-orthogonality between subcarrier. So, MAI in multipath fading channel is more than AWGN channel due to ICI.

\section{Code-based parallel interference cancellation technique (code-PIC)}

As discussed in Section 4, there are two groups of users, $B_{1}$ and $B_{2}$, based on data rates where $U_{1} \in B_{1}, U_{2,3,4,5} \in$ $B_{2}$ and $U_{2} \cap U_{3} \cap U_{4} \cap U_{5}=\varphi$. The users of $B_{1}$ group utilize $N$ available subcarriers, and $B_{2}$ users employ alternate odd/even subcarrier. Users in $B_{2}$ group are assigned pseudo-orthogonal $\mathrm{CI}$ ( $\mathrm{PO}-\mathrm{CI})$ codes such that cross-correlation between users from $B_{1}$ and $B_{2}$ group is low. This results in reduced MAI between users.

The estimated interference is cancelled out using a code-based PIC (Code-PIC) scheme. Steps involved in Code-PIC scheme is described next with a simplified structure shown in Figure 2.

\subsection{Steps involved in Code-PIC scheme}

Received signal $r(t)$ is projected onto $N$ orthogonal subcarriers. The initial estimates of all users $(1 \geq k \geq 3 N)$ are obtained with single-user detector (SUD). In multistage iterative receiver, all users from a selected group are detected first. After that, all users from the next groups are selected. In Code-PIC, MAI is reduced using the following steps at a given iteration:

step 1: At the first stage of iterative receiver, the group of desired user (say $j$ th user) is identified.

step 2: If the desired user belongs to $B_{2}$ group (LoDR), then signal components for $B_{1}$ users are reconstructed and projected onto $N$ subcarriers. Now, the MAI due to all $B_{1}$ users is estimated on $i$ th subcarrier. Estimated interference is subtracted from the received signal. After that, steps 3 and 4 are performed.

OR

If the desired user group is $B_{1}$, then to obtain the decision on odd subcarrier, reconstructed signals of $U_{2}$ and $U_{3}$ are considered; otherwise, for even subcarrier operation, reconstructed signal of $U_{4}$ and $U_{5}$ users are projected on $i$ th subcarrier. MAI due to $B_{2}$ group is estimated and subtracted from the received signal component at subcarrier level. Step 4 is performed for all users of $B_{1}$ group.

step 3: The subcarrier set (ith subcarrier) of $j$ th user is identified. If the subcarrier set is odd subcarrier, then signal components due to $U_{2}$ and $U_{3}$ set are reconstructed; otherwise, $U_{4}$ and $U_{5}$ users are considered. Then, the code pattern (ODD CI or EVEN CI) of $j$ th user is also detected. If the code pattern is ODD CI, then reconstructed signal components of $U_{3}$ or $U_{5}$ user sets (depends on which user set is selected based on $i$ th subcarrier set) are projected on the $i$ th subcarrier; otherwise $U_{2}$ or $U_{4}$ user sets are projected. MAI due to projected user sets is estimated and subtracted from the received signal.

step 4: The received signal component consists of users of only $j$ th user set. The interference due to other users of $j$ th user set is estimated and subtracted to obtain improved decision via decision combiner for $j$ th user. This step is repeated for all users of $j$ th user set.

These steps are performed for all users of the selected group. Next, we discuss the decoding of $B_{1}$ and $B_{2}$ users in 5.2 and 5.3 subsection, respectively. 


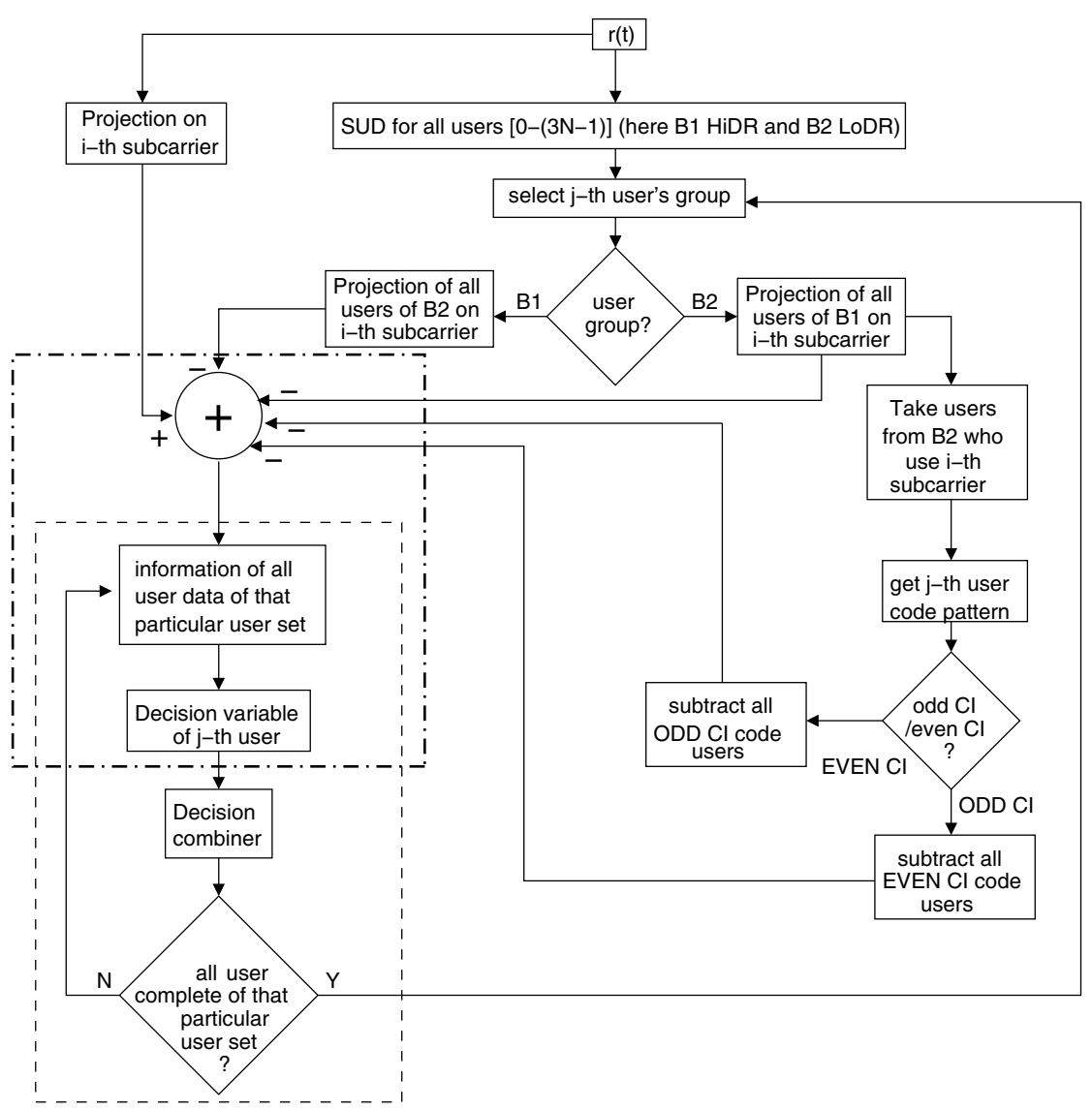

Figure 2 Code-PIC algorithm.

\subsection{Decoding of $B_{1}$ users}

For a given desired user from $B_{1}$ group, MAI is caused due to all users from $B_{1}$ group and the users of $B_{2}$ who use same subcarrier of $B_{1}$ group. The estimated MAI of $k$ th user due to other $(K-1)$ users at 'iter' iteration stage $\left(\hat{\mathbf{I}}_{k}^{i t e r}\right)$ may be expressed as

$$
\begin{aligned}
\hat{\mathbf{I}}_{k}^{\text {iter }} & =\Re\left[\sum_{i=0}^{N-1} \sum_{m=1, m \neq k}^{N} \sqrt{\frac{2 E_{b}}{N_{0}}} \hat{a}_{m}^{i t e r-1} e^{j i\left(\Delta \theta_{m}-\Delta \theta_{k}\right)}\right. \\
& +\sum_{i=0 \forall i=\text { odd }}^{N-1}\left(\sum_{m=N+1}^{3 N / 2} \sqrt{\frac{2 E_{b}}{N_{0}}} \hat{a}_{m}^{i t e r-1} e^{j i\left(\Delta \theta_{m}-\Delta \theta_{k}\right)}\right. \\
& \left.+\sum_{m=(3 N / 2)+1}^{2 N} \sqrt{\frac{2 E_{b}}{N_{0}}} \hat{a}_{m}^{i t e r-1} e^{j(i+1)\left(\Delta \theta_{m}-\Delta \theta_{k}\right)}\right) \\
& +\sum_{i=0 \forall i=e v e n}^{N-1}\left(\sum_{m=2 N+1}^{5 N / 2} \sqrt{\frac{2 E_{b}}{N_{0}}} \hat{a}_{m}^{i t e r-1} e^{j i\left(\Delta \theta_{m}-\Delta \theta_{k}\right)}\right. \\
& \left.\left.+\sum_{m=(5 N / 2)+1}^{3 N} \sqrt{\frac{2 E_{b}}{N_{0}}} \hat{a}_{m}^{i t e r-1} e^{j(i+1)\left(\Delta \theta_{m}-\Delta \theta_{k}\right)}\right)\right]
\end{aligned}
$$

and

$$
\hat{I}_{k\left(U_{1}\right)}^{i t e r}=\hat{I}_{k\left(U_{1}, U_{1}\right)}^{i t e r}+\hat{I}_{k\left(U_{1}, U_{2}\right)}^{i t e r}+\hat{I}_{k\left(U_{1}, U_{3}\right)}^{i t e r}+\hat{I}_{k\left(U_{1}, U_{4}\right)}^{i t e r}+\hat{I}_{k\left(U_{1}, U_{5}\right)}^{i t e r}
$$

where $\hat{a}_{k}^{i t e r}, \hat{I}_{k\left(U_{i}\right)}^{i t e r}$ and $\hat{I}_{k\left(U_{i}, U_{j}\right)}^{i t e r}$ are the estimated data of $k$ th user, total estimated MAI for $U_{i}$ user set and MAI due to $U_{j}$ user set for the $U_{i}$ user set, respectively, at 'iter' iteration stage. We assumed that HDR users transmit data at 'q' times higher than LoDR users. While calculating $\hat{I}_{k\left(U_{1}\right)}^{i t e r}$ for $n$th bit, $\hat{I}_{k\left(U_{1}, U_{i}\right)}^{i t e r},(i=2,3,4$, 5) remains same for taking the decision of all consecutive 'q' number of bits. So, time and complexities become less in Code-PIC technique. The major drawback of this type of technique is that if one of the bits of LoDR is wrongly estimated, then it can effect 'q' number of HDR bits. Error propagation can be minimized if hard decision is replaced by soft decision of received data bits $[7,10,17]$. In the last stage of iteration, the final decision is made by hard detector, $\hat{a}_{k}=\operatorname{sgn}\left\{\mathbf{Y}_{k}-\hat{\mathbf{I}}_{k}^{i t e r}\right\}$. 


\subsection{Decoding of $B_{2}$ users}

Let us take $U_{2}$ user set as one of the desired user set of $B_{2}$ group. Only odd subcarriers of the available subcarriers are used by $U_{2}$ set. So, the users who use odd subcarrier create interference on $U_{2}$ set. All $B_{1}$ users are non-orthogonal to set $B_{2}$ users. Interference due to HDR users can be written as

$$
\hat{I}_{k\left(U_{2}, U_{1}\right)}^{\text {ter }}=\Re\left[\sum_{i=0 \forall i=o d d}^{N-1} \sum_{m \in B_{1}} \sqrt{\frac{2 E_{b}}{N_{0}}} \hat{a}_{m}^{\text {iter-1 }} \exp \left[j\left(i\left(\Delta \theta_{m}-\Delta \theta_{k}\right)\right)\right]\right]
$$

In $B_{2}$ group, only $U_{2}, U_{3}$ users utilize odd subcarriers. There is no interference due to $U_{4}, U_{5}$, assuming proper orthogonality maintained in subcarrier. $\hat{I}_{k\left(U_{2}\right)}^{i t e r}$ can be written as

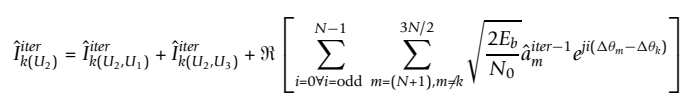

This proper estimation and subtraction of MAI from the received signal improves the system performance. MAI experienced by other users set can be obtained in similar way.

\section{Simulation results}

This section demonstrates the BER performance comparison of BPSK-modulated synchronous CI/MC-CDMA system with Block-PIC, Sub-PIC and Code-PIC at different signal-to-noise ratios (SNR) using Monte Carlo simulations in MATLAB. Both hard and soft decisions of received data bits are used to estimate the MAI. Perfect channel estimation and synchronization are assumed at the receiver. No forward error correcting code is employed for data transmission. For multipath frequency selective channel, we have assumed 4-fold Rayleigh fading [21]. It is also assumed that HDR users transmit data at 4 times higher than LoDR users. In the next subsection, results over AWGN channel are presented and then the results over Rayleigh fading channel are reported.

\subsection{AWGN channel}

Figure 3 illustrates the performance of SDCode-PIC technique for 2.5 user multirate system with 64 HDR users and 96 LoDR users. Number of subcarriers $(N)$ is 64. From the figure, it is clear that BER performance improves by increasing the number of iterations. The estimated MAI becomes closer to actual MAI as number of iterations increases. So, the residual part of MAI $\left(\mathbf{I}_{k}-\hat{\mathbf{I}}_{k}^{\text {ter }}\right)$ becomes less. Subtraction of estimated MAI results in the improvement in BER performance. After 5th stage of iteration, a BER of $1.3 \mathrm{e}-03$ is obtained at 10 $\mathrm{dB}$ SNR. Bit error probability of $6.7 \mathrm{e}-04$ is observed after 8th iteration, at same SNR. After a certain number

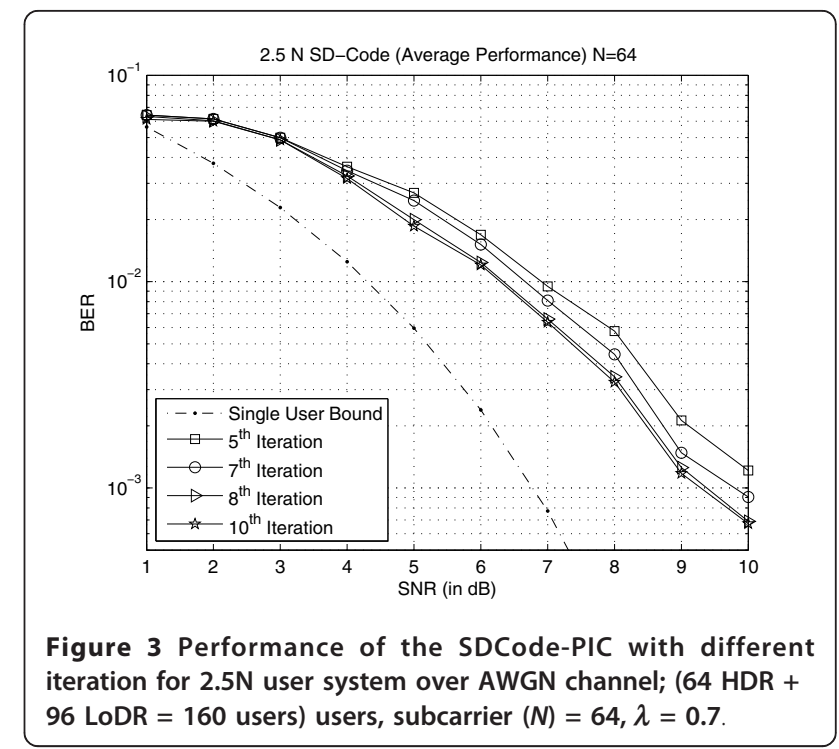

of iterations, the residual interference cannot be removed further. So, BER performance remains almost same for higher number of iterations. From the simulation, the performances of 8th and 10th stages are almost same. So, for $2.5 \mathrm{~N}$ user multirate system, the number of iterations is fixed at 8 without increasing latency and complexities involved in higher stage of iterations.

The performance comparison of SDCode-PIC and SDSub-PIC scheme is evaluated in Figure 4 for $2.5 \mathrm{~N}$ multirate system ( $N$ HDR users and 1.5N LoDR users). A total of 160 users (64 HDR + 96 LoDR) are transmitting data at two different data rates over AWGN channel. In SDSub-PIC, estimation of the interference for desired user is done without considering interference

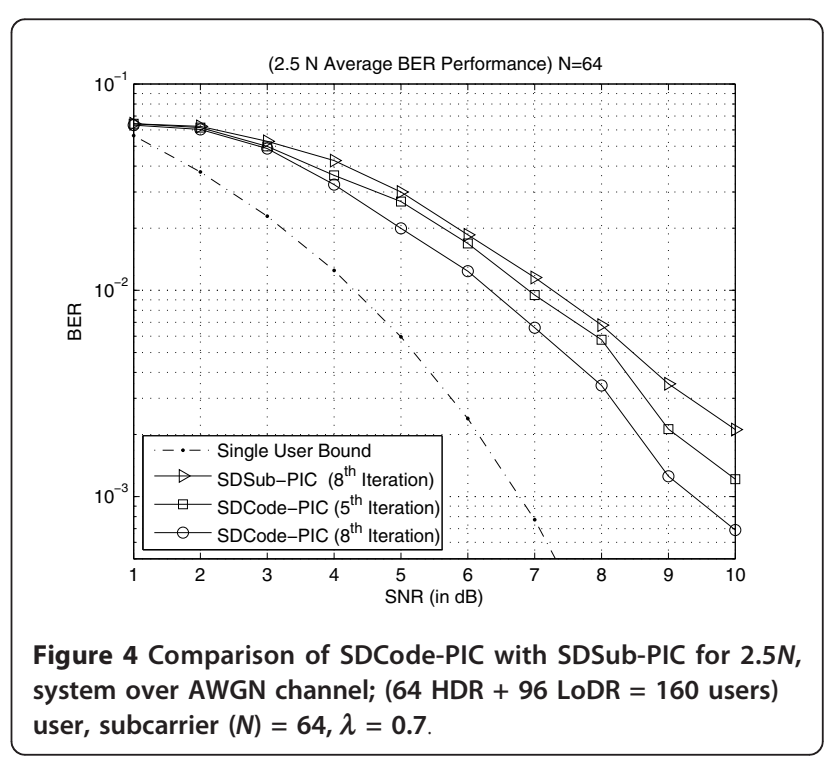


from other user group. So, large number of iteration stages is required to cancel interference to achieve allowable BER. In SDCode-PIC, the interference is estimated based on the knowledge of desired user group and interfering user group. So, the improved estimation ensures less number of iteration to get same BER performance or even better than SDSub PIC. From the figure, it is clear that the performance of SDCode-PIC after 5th stage is better than that of the 8th stage of SDSub-PIC over an AWGN channel. A SNR gain of 1.5 $\mathrm{dB}$ is obtained in SDCode-PIC compared to SDSub-PIC at a BER of 2e-03 after 8th stage of iteration.

In Figure 5, the results are reported for evaluating the effect of adding users more than $N(K>N)$, i.e., overloading in multirate $\mathrm{CI} / \mathrm{MC}$-CDMA system. The number of high data rate (HDR) users is fixed at 64 . The interference effect on high data rate users due to LoDR group is observed in this figure. For 96 LoDR users (1.5N LoDR), the interference due to LoDR is more than 76 LoDR $(1.2 \mathrm{~N}$ LoDR) user system. The average BER of $2.5 \mathrm{~N}(1 \mathrm{~N} \mathrm{HDR}+$ $1.5 \mathrm{~N}$ LoDR) and $2.2 \mathrm{~N}(1 \mathrm{~N} \mathrm{HDR}+1.2 \mathrm{~N}$ LoDR) user multirate systems are $6.2 \mathrm{e}-04$ and $4.5 \mathrm{e}-04$, respectively, at $10 \mathrm{~dB}$ SNR using SDCode-PIC after 8th iteration over AWGN. System is also tested with 70 LoDR $(1.1 N)$ users with subcarrier $(N)=64$. At $10 \mathrm{~dB}$ SNR, the BER reduces to 3e-04 after same iteration over an AWGN channel. The degradation in SNR is $2.3 \mathrm{~dB}$ compared to single user bound over AWGN channel at a BER of 3e-04. A SNR gain of 0.8 $\mathrm{dB}$ is obtained in $2.1 \mathrm{~N}$ system compared to $2.2 \mathrm{~N}$ user system at a BER of 6e-04. The gain in SNR is $1.3 \mathrm{~dB}$ in $2.1 \mathrm{~N}$ user system compared to $2.5 \mathrm{~N}$ user system at $7 \mathrm{e}-04 \mathrm{BER}$.

\subsection{Rayleigh fading channel}

In Figure 6, the performance of Code-PIC is compared with Block-PIC [24] and Sub-PIC [25] for $2 N$ system

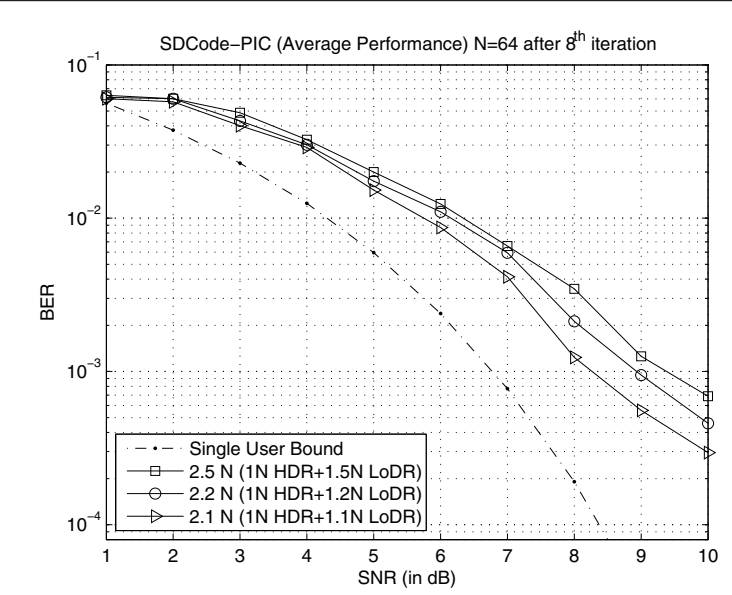

Figure 5 Different loading in SDCode-PIC with different SNR (in dB) value over AWGN channel; subcarrier $(N)=64$, and $\lambda=0.7$.

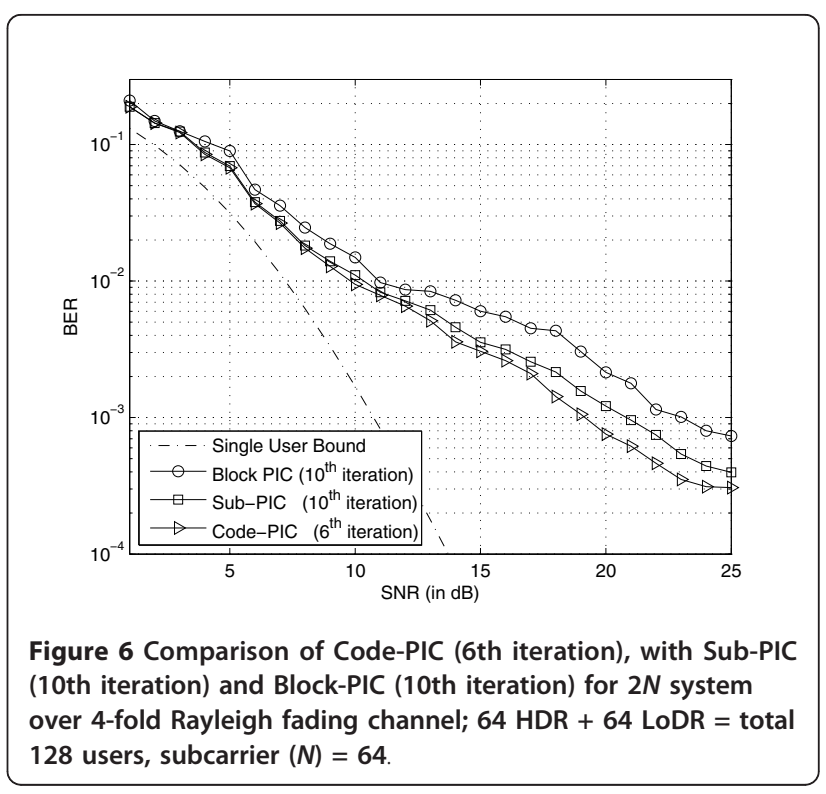

with hard decisions. 64 (1N) HDR users, 32 LoDR $(N / 2)$ users (using odd subcarrier) and 32 LoDR (N/2) users (using even subcarrier), i.e., a total of 128 users transmit data simultaneously. After 10th stage of iteration, a BER of 7.3e-04 is obtained at $25 \mathrm{~dB}$ SNR with Block-PIC. In Sub-PIC, a BER of $4 \mathrm{e}-04$ is observed at $25 \mathrm{~dB}$ SNR. But, in Code-PIC, only after 6th iteration, BER of 3e-04 is observed. From the figure, it is clear that Code-PIC provides a performance gain of about $4 \mathrm{~dB}$ and $2 \mathrm{~dB}$ compared to Block-PIC and Sub-PIC, respectively, at a BER of $1 \mathrm{e}-03$ with reduced number of iterations.

Figure 7 illustrates the performance comparison between three soft decision-based PIC schemes. At 25 dB SNR, a BER of 5.6e-05 is obtained using SDCode-

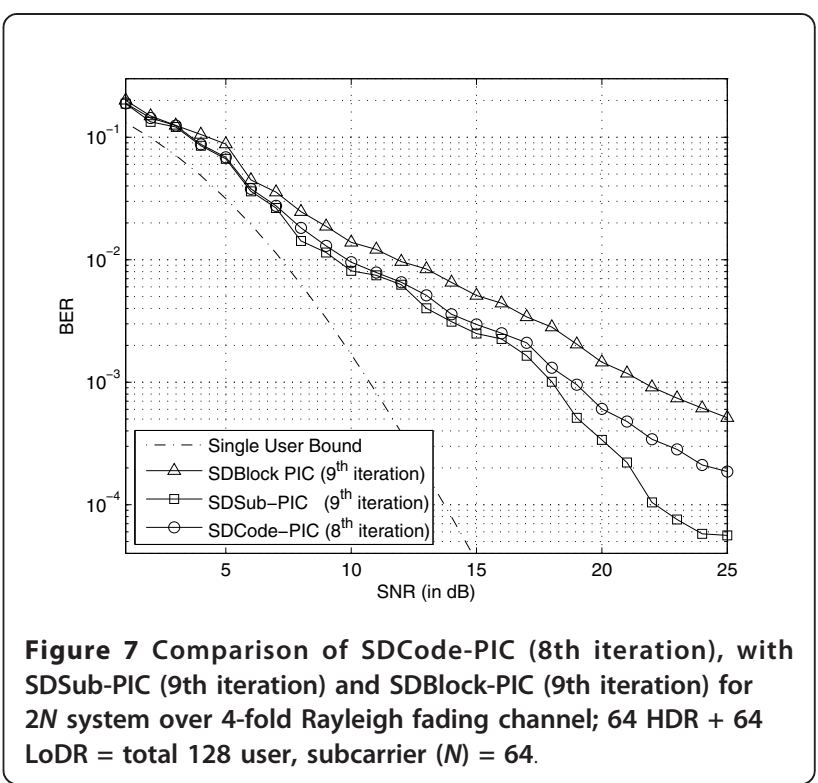


Table 1 Complexity per iteration $\left(\mathcal{C}_{P I C}\right)$ for four PIC schemes

\begin{tabular}{ll}
\hline PIC Scheme & Complexity \\
\hline Conventional & $N\left[\left(K_{1}+K_{2}+1\right) N+1\right]\left(K_{1}+K_{2}\right)$ \\
PIC & $N\left[\left(\log \left(K_{1}+K_{2}\right)-1\right) N+1\right] \log \left(K_{1}+K_{2}\right)$ \\
Block-PIC & $+N\left[\left(K_{1}+K_{2}-\log \left(K_{1}+K_{2}\right)-1\right) N+1\right]\left(K_{1}+K_{2}-\log \left(K_{1}+\right.\right.$ \\
& $\left.\left.K_{2}\right)\right)$ \\
Sub-PIC & {$\left[N\left(K_{1}+K_{2}\right)+1\right]\left(\frac{K_{1}}{4}+K_{2}\right)+\left[N\left(K_{1}+1\right)+1\right] \frac{3 K_{1}}{4}$} \\
Code-PIC & {$\left[N\left(K_{1}+K_{2}\right)+1\right] \frac{K_{1}}{4}+\left[N\left(K_{1}+1\right)+1\right] \frac{3 K_{1}}{4}+\left[N\left(K_{1}+\frac{K_{2}}{4}-1\right)+1\right] K_{2}$} \\
\hline
\end{tabular}

PIC after 8th iteration compared to $5 \mathrm{e}-04$ and $2 \mathrm{e}-04$ for SDBlock-PIC and SDSub-PIC, respectively, after 9th iteration. From the result, it is clear that soft decisionbased Code-PIC (SDCode-PIC) performs significantly better than soft decision-based Sub-PIC (SDSub-PIC) [30] and soft decision based Block-PIC (SDBlock-PIC) with less number of iterations. From the figure, it is clear that SDCode-PIC performs better than SDBlockPIC and SDSub-PIC with reduced complexity.

It has been observed through simulations that for a given BER of about 1e-03, Code-PIC requires 4 iterations, while Block-PIC and Sub-PIC require 8 and 7 iterations, respectively, for $2 \mathrm{~N}$ system. Also from Figures 6 and 7, it is observed that Code-PIC requires less number of iterations and hence results in reduced latency.

\section{Complexity comparison}

This section evaluates the computational complexities of conventional PIC [24], Block-PIC [24], Sub-PIC [25] and Code-PIC for multirate CI/MC-CDMA system over AWGN channel. Computational complexity per bit period of PIC algorithm is computed in terms of number of HDR users $\left(K_{1}\right)$, number of LoDR users $\left(K_{2}\right)$, number of available subcarriers $(N)$ and number of iterations (num_iter) [31]. We define the complexity unit as one real multiplication or one signed addition. More complex operation like division is considered as multiplication operation [32]. It is also assumed that the $\operatorname{sgn}($. operation and binary comparison require no additional computational complexity [32].

In multirate CI/MC-CDMA, it is assumed that there are $K_{1}$ HDR users and $K_{2}$ LoDR users $\left(K_{1}+K_{2} \geq 3 N\right)$. The number of LoDR users in $U_{i},(i=2,3,4,5)$ set equals to
$K_{2} / 4$. In a given bit period, the total computational complexity of multistage PIC detector can be expressed as

$$
\mathcal{C}_{\mathrm{TOTAL}}=\text { num_iter } \times \mathcal{C}_{\mathrm{PIC}}+\mathcal{C}_{x}
$$

where $\mathcal{C}_{\text {PIC }}$ is the complexity of one iteration for the hard decision PIC, and $\mathcal{C}_{x}$ is additional computation required for soft decision PIC technique (equals to zero if only hard decision is used). Table 1 shows the $\mathcal{C}_{\text {PIC }}$ of one iteration for the four PIC schemes.

In conventional PIC, computation complexity is $N\left[K_{1}\right.$ $\left.\left.+K_{2}+1\right) N+1\right]\left(K_{1}+K_{2}\right)$ per iteration for $\left(K_{1}+K_{2}\right)$ users. From Table 2 and Figure 8 , it is observed that complexity of Block-PIC is almost same as conventional PIC, which is also reported in [24]. In Sub-PIC, MAI is estimated and subtracted at subcarrier level. So, computational complexity is reduced compared to Block-PIC and conventional PIC schemes. In Code-PIC, computation required to estimate the MAI is significantly reduced by the proper selection of the interfering user sets. This further simplifies the subtraction of MAI. Hence, the computation complexity CCCC is significantly less for Code-PIC compared to other schemes. It is also observed from Table 2 that for a given system load, complexity of Code-PIC is significantly less than conventional PIC and Block-PIC. Further, it is observed from Figure 8 that the complexity of Code-PIC is comparable to Sub-PIC up to a system load of about $1.5 \mathrm{~N}$ and for higher loads Code-PIC outperforms Sub-PIC.

\section{Conclusion}

In this paper, Code-PIC scheme is introduced for multirate CI/MC-CDMA system. The performance is compared with Block-PIC and Sub-PIC with hard and soft estimates of received data bits over AWGN and frequency selective Rayleigh fading channels. The proposed scheme provides significant performance improvement with less complexity and reduced latency compared to PIC schemes like Block-PIC and Sub-PIC. In frequency selective channel for $2 N$ multirate system $(N=64)$, SDCode-PIC ensures SNR gain of $6 \mathrm{~dB}$ and $2 \mathrm{~dB}$ compared to SDBlock-PIC and SDSub-PIC, respectively, at a BER of 5e-04. From the results, we conclude that CodePIC is a powerful technique to reduce MAI for multirate $\mathrm{CI} / \mathrm{MC}$-CDMA system over frequency selective channel with overloaded condition. It will be interesting to

Table $2\left(\mathcal{C}_{P I C}\right)$ of the 1 st iteration for different PIC schemes

\begin{tabular}{llllll}
\hline PIC & \multicolumn{5}{c}{ System load } \\
\hline & $\mathbf{1 N}$ & $\mathbf{1 . 5 N}$ & $\mathbf{2 N}$ & $\mathbf{2 . 2 5 N}$ & $\mathbf{2 . 5 N}$ \\
& $\mathbf{1 N}$ HDR & $\mathbf{1 N}$ HDR+0.50N LoDR & $\mathbf{1 N}$ HDR+1N LoDR & $\mathbf{1 N}$ HDR+1.25N LoDR & $\mathbf{1 N}$ HDR+1.5N LoDR \\
\hline Conventional PIC & 16519168 & 37361664 & 66592768 & 84354048 & 104212480 \\
Block-PIC & 22133254 & 32088263 & 59484359 & 76328135 & 95269063 \\
Sub-PIC & 363600 & 494688 & 855168 & 1084560 & 1346720 \\
Code-PIC & 265280 & 445536 & 658560 & 777360 & 904352 \\
\hline
\end{tabular}




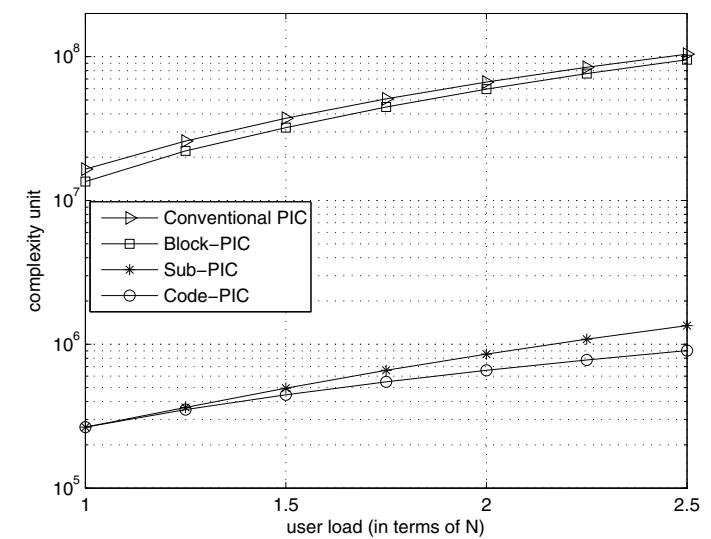

Figure 8 Complexity for 1 st iteration of different PIC schemes at different user load with 64 subcarrier $(N)$; number of HDR users is fixed at $64(1 N)$, number of LoDR user is varied from 6 $(0.1 N)$ to $64(1 N)$.

evaluate the performance of this scheme under imperfect timing and frequency synchronization over nonideal channel conditions. The SNR penalty can be reduced further by using suitable error correcting codes.

\section{Acknowledgements}

The authors would like to thank the anonymous reviewers for their constructive comments.

\section{Competing interests}

The authors declare that they have no competing interests.

Received: 13 April 2011 Accepted: 2 November 2011

Published: 2 November 2011

\section{References}

1. S Verdu, Multiuser Detection (Cambridge: Cambridge University Press, 1998)

2. A Kocian, B Fleury, EM -based joint data detection and channel estimation of DS- CDMA signals. IEEE Transactions on Communications 51(10), 1709-1720 (2003). doi:10.1109/TCOMM.2003.818091

3. G Woodward, R Ratasuk, M Honig, P Rapajic, Minimum mean-squared error multiuser decision-feedback detectors for DS-CDMA. IEEE Transactions on Communications 50(12), 2104-2112 (2002). doi:10.1109/ TCOMM.2002.806550

4. A Kapur, M Varanasi, Multiuser detection for overloaded CDMA systems. IEEE Transactions on Information Theory 49(7), 1728-1742 (2003). doi:10.1109/TIT.2003.813562

5. P Patel, J Holtzman, Analysis of a simple successive interference cancellation scheme in a DS/CDMA system. IEEE Journal on Selected Areas in Communications 12(5), 796-807 (1994). doi:10.1109/49.298053

6. D Divsalar, M Simon, D Raphaeli, A new approach to parallel interference cancellation for CDMA, in IEEE GLOBECOM 3, 1452-1457 (1996)

7. D Divsalar, M Simon, D Raphaeli, Improved parallel interference cancellation for CDMA. IEEE Transactions on Communications 46(2), 258-268 (1998). doi:10.1109/26.659484

8. M Mostofa, K Howlader, B Woerner, Iterative interference cancellation and decoding using a soft cancellation factor for DS-CDMA, in IEEE VTC. 3, 2076-2080 (2000)

9. RR Muller, JB Huber, Iterated Soft-Decision Interference Cancellation for CDMA, in Broadband Wireless Communications, ed. by Luise and Pupolin (Springer, 1998), pp. 110-115
10. W Zha, S Blostein, Soft-decision multistage multiuser interference cancellation. IEEE Transactions on Vehicular Technology 52(2), 380-389 (2003)

11. W Hamouda, P McLane, A fast adaptive algorithm for MMSE receivers in DS- CDMA systems. IEEE Signal Processing Letters 11(2), 86-89 (2004). doi:10.1109/LSP.2003.819855

12. R de Lamare, R Sampaio-Neto, A Hjorungnes, Joint iterative interference cancellation and parameter estimation for CDMA systems. IEEE Communications Letters 11(12), 916-918 (2007)

13. R de Lamare, R Sampaio-Neto, Minimum Mean-Squared Error Iterative Successive Parallel Arbitrated Decision Feedback Detectors for DS-CDMA Systems. IEEE Transactions on Communications 56(5), 778-789 (2008)

14. N Correal, R Buehrer, B Woerner, Improved CDMA performance through bias reduction for parallel interference cancellation, in IEEE PIMRC 97, 565-569 (1997)

15. P Renucci, B Woerner, Optimisation of soft interference cancellation for DSCDMA. Electronics Letters 34(8), 731-733 (1998). doi:10.1049/el:19980512

16. D Djonin, V Bhargava, New results on low complexity detectors for oversaturated CDMA systems, in IEEE GLOBECOM. 2, 846-850 (2001)

17. F Vanhaverbeke, M Moeneclaey, H Sari, Increasing CDMA capacity using multiple orthogonal spreading sequence sets and successive interference cancellation, in IEEE International Conference on Communications. 3, 1516-1520 (2002)

18. P Kumar, S Chakrabarti, A New Overloading Scheme for Cellular DS-CDMA using Orthogonal Gold Codes. IEEE VTC, 1042-1046 (2008)

19. P Kumar, S Chakrabarti, An Analytical Model of Iterative Interference Cancellation Receiver for Orthogonal/Orthogonal Overloaded DS-CDMA System. Springer's International Journal of Wireless Information Networks 17, 64-72 (2010). doi:10.1007/s10776-010-0112-2

20. YP Tu, WH Fang, HY Lu, YT Chen, Iterative Multiuser Detection with Soft Interference Cancellation for Multirate MC-CDMA Systems. IEEE VTC, 708-712 (2008)

21. B Natarajan, C Nassar, S Shattil, M Michelini, Z Wu, High-performance MCCDMA via carrier interferometry codes. IEEE Transactions on Vehicular Technology 50(6), 1344-1353 (2001). doi:10.1109/25.966567

22. D Wiegandt, Z Wu, C Nassar, High-throughput, high-performance OFDM via pseudo-orthogonal carrier interferometry spreading codes. IEEE Transactions on Communications 51(7), 1123-1134 (2003). doi:10.1109/ TCOMM.2003.814196

23. M Michelini, S Hijazi, C Nassar, Z Wu, Spectral sharing across 2G-3G systems, in Conference Record of the Thirty-Seventh Asilomar Conference on Signals, Systems and Computers, 2003. 1, 13-17 (2003)

24. V Thippavajjula, B Natarajan, Parallel interference cancellation techniques for synchronous carrier interferometry/MC-CDMA uplink $4 \mathrm{G}$ cellular systems, in IEEE VTC. 1, 399-403 (2004)

25. SP Maity, M Mukherjee, Subcarrier PIC Scheme for High Capacity CI/MCCDMA System with Variable Data Rates. IEEE Mobile WiMAX Symposium 0 135-140 (2009)

26. W Xu, LB Milstein, Performance of multicarrier DS-CDMA systems in the presence of correlated fading, in IEEE VTC. 3, 2050-2054 (1997)

27. A Akansu, M Tazebay, M Medley, P Das, Wavelet and subband transforms: fundamentals and communication applications. IEEE Communications Magazine 35(12), 104-115 (1997). doi:10.1109/35.642839

28. Y Lee, N Kim, H Park, Performance of MC-CDMA Systems With MMSEC Over Rayleigh Fading Channels. IEEE Transactions on Vehicular Technology 59(6), 3126-3130 (2010)

29. M Mukheriee, P Kumar, A New Code Allocation Algorithm for Multirate Cl/ MC-CDMA System. IEEE Conference on Communication Systems and Networks (COMSNETS), 1-6 (2011)

30. M Mukherjee, P Kumar, A New Iterative Soft Decision Subcarrier PIC scheme for CI/MC-CDMA system. IEEE PIMRC 2010 Fundamentals and PHY (PIMRC 2010 Track 1), 767-772 (2010)

31. R Buehrer, N Correal-Mendoza, B Woerner, A simulation comparison of multiuser receivers for cellular CDMA. IEEE Transactions on Vehicular Technology 49(4), 1065-1085 (2000). doi:10.1109/25.875213

32. IDR Brown, Multistage parallel interference cancellation: convergence behavior and improved performance through limit cycle mitigation. IEEE Transactions on Signal Processing 53, 283-294 (2005)

doi:10.1186/1687-1499-2011-155

Cite this article as: Mukherjee and Kumar: A novel code-based iterative PIC scheme for multirate CI/MC-CDMA communication. EURASIP Journal on Wireless Communications and Networking 2011 2011:155. 